\title{
Towards Amblyopia Therapy Using Mixed Reality Technology
}

\author{
Adam Nowak, Mikołaj Woźniak, Michał Pieprzowski, Andrzej Romanowski \\ Institute of Applied Computer Science \\ Lodz University of Technology \\ 90-924 Lodz, Poland \\ email: 203151@edu.p.lodz.pl, mikolaj@pawelwozniak.eu,210893@edu.p.lodz.pl, androm@iis.p.lodz.pl
}

\begin{abstract}
This paper presents an approach towards aiding the rehabilitation exercises in amblyopia care using mixed reality technology. The Lazy Eye Syndrome is tackled here through an interactive holographic application implemented on Microsoft HoloLens device. It provides an entertaining way for the handicapped eye workout as it is based on a simple game of skill. The game is designed in a way that the majority of awarerequiring objects and events are displayed for the cured eye only, remaining the other eye responsible for background and additional information perception. Such disproportion forces an increased activity of the lazy eye, which is to perform more movements and impose the brain to process the sight more extensively. The proposed prototype is an extension of a novel approach towards treating amblyopia, employing software-based stimulation techniques, which could be easily adapted to various age and ability correlated needs of the user, with minimal requirements regarding the exercise setting and preparation.
\end{abstract}

\section{INTRODUCTION}

$\mathbf{T}$ HE amblyopia, often referred as the lazy eye syndrome is one of the most frequently diagnosed vision disorders among children. The cause of this affliction lays in disrupted cooperation between the eye and the brain. The brain tends to prefer the other eye for visual perception, therefore one of the eyes performs significantly less movements and activity, which causes further decrease of vision in the handicapped eye [1].

The lazy eye syndrome, if not properly treated during childhood, is likely to maintain an issue during adulthood. Such disease prevents an individual from obtaining professional driving licenses, which significantly limits the job opportunities. Moreover, the improper balance of eye activity might increase the risk of other eye injuries and diseases for the healthy eye [2].

The traditional approach of treating amblyopia is to address children only, as the therapy provides best efficiency during the so-called window of visual cortex development. The most common method prescribes putting a patch over the properly working eye and performing high eye-awareness requiring activities, forcing the brain to perform with the disabled eye more intensely. Such approach brings satisfactory results when applied to children in the age between 5 and 8 years old. However, the method is being widely discussed, as it employs highly artificial conditions of eye operation, with no regard of the stereoscopic vision.
Furthermore, noncompliance to wearing the patch (fulltime or part-time, accordingly to the diagnosis) drives the treatment to failure. Therefore, the commitment required from the children, as well as their parents - as the proper supervision is necessary for that method, is significantly affecting daily life. Moreover, wearing a patch may cause social anxiety in the peer group and pose a huge challenge in performing some of school and educational tasks.

Thompson et. al. concerned about adult treatment, where the main problem seemed to be the lack of brain plasticity. Experiment with repetitive Transcranial Magnetic Stimulation proved that contrast sensitivity may be improved in the amblyopic eye by regular 10 minutes sessions with rTMS. This is the evidence that neuronal plasticity is not fully attenuated in adulthood. [3]. Further, it is suggested that amblyopia has a binocular nature, being a disrupt of analysing the signals perceived by both eyes. Having provided extended time of viewing and different contrast for each eye, the improvement of vision is noticed [4]. Therefore, the binocular manner of exercising may be perceived as most favorable and beneficial.

In this paper, we would like to propose low-commitment approach towards exercising the amblyopic eye, suitable for both children and adults (yet dedicated mainly to youngster users) concerning workout sessions in home setting.

\section{RELATED WORK}

Multiple attempts have been made towards alleviating the constraints of traditional amblyopia treatment. One of the most promising approaches is to employ video games for stimulating the amblyopic eye through display design, with no constraint of losing the stereoscopic vision. Li et al. [5] prove that playing video games utilizing the lazy eye significantly develop the fundamental vision functions of the patient [5]. The improvements to a different extent were found for both visual and positional acuity, spatial attention, and the most significant for stereopsis. Cross-over study shown that occlusion approach cannot provide improvements in all those areas.

Those conclusions inspired further development of a tool which could serve as the exercise equipment for game-based treatment. Eastgate et. al. [6] proposed an integrated system, employing VR technology, enabling to practise through 
playing various $2 \mathrm{D}$ and $3 \mathrm{D}$ games, stimulating the vision in different manners, as well as implemented a control view for the clinician. The binocular system provided an extensive setup for game-based lazy-eye treatment, while its high-complexity and ambient requirements made it available only for clinical use, through organised sessions. One of recent reports related to use of Oculus VR technology for adult patients is [7]. Such manner of operation does not solve the high-commitment constraint, as forcing daily visits to the clinic pose additional challenge to the patient's everyday routine.

Bringing the therapy to patient's home seemed to be an ultimate goal in a way to reducing the commitment and obstacles present in the process. Birch et. al.[8] tried to answer this need by proposing a binocular system based on Apple iPad applications. This approach enabled users to exercise in home setting, while being constrained with limited support of optic equipment available for iPad. The study has shown that playing the games using binoculars brought favorable results while being combined with traditional patch-based treatment. Gargantini et al. [9] took another endeavour towards providing affordable solution of home-exercising of the lazy eye syndrome, using Google Cardboard and the smartphone application. This solution differs the level of details displayed in each eye, forcing the amblyopic one to process more information than the healthy eye. The application also supported additional features for doctors. The main advantage of this solution is low-cost implementation for commonly used devices.

A commercial approach to bringing the VR treatment experience to patient's home has been made by Vivid Vision [10]. This solution employed commercially accessible technologies such as Oculus Rift, HTC Vive, Samsung Gear VR. However, even the end-user VR solutions pose high requirements towards the setting of playing, as well as disabling the awareness of any outer factors. In terms of children usage, the setup requires parental supervision, as the risk of undesired action is decent (eg. walking into furniture); see Fig 1. Therefore, we can establish the need for a solution which possess the advantages of VR projection while remaining ambient awareness of the performed activity. Mezad-Koursh et. al. [11] prove the necessity of daily exercising in successful treatment through pilot study of home use of a similar system, based on watching animated television using binoculars. The regular training enables improvements of the amblyopic eye activity even for children older than the assumed visual cortex development window.

Our attempt aims to bring the convenience of mobile solution [8], supported with high eye-involvement and immersive experience of the [6].

\section{HOLOLENS IMPLEMENTATION}

The proposed solution employs the Microsoft HoloLens, which is the leading commercial equipment for augmented reality projections. Due to AR approach, the displayed projection can be easily adapted to the ambient conditions, both in terms of visuals and their color contrasts, but also through real-object recognition and automated space-aware algorithm, so the artifacts will not be displayed on the physical obstacles, which becomes a major issue concerning the solution might be used by children.

Unlike the traditional, full-covering VR technology, the HoloLens offers undisturbed perception of the surrounding. As a consequence, the risk of undesired physiological reactions is significantly lower than for full-covering VR [12]. What is more, the experience of full immersion into VR activities is an additional challenge to the brain, which is naturally opposing the perception through display-equipped eyes and other senses. This effect is especially not recommended to the children [13]. Such effects are not observed for augmented reality displays, as the frame of reference is not altered. Therefore, it is more advantageous not to distract the brain with the senses' counteractions and maintain focus on extended activity of the amblyopic eye. The lack of harmful side effects of using the system is especially important due to the specific of the amblyopia curing procedure - the eye shall be forced to operate with enhanced activity for longer periods of time.

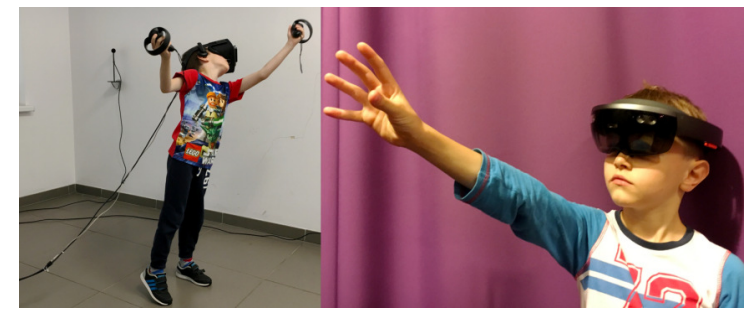

Fig. 1. 8yo user operating VR app (left) and the HoloLens app (right). The AR game is being displayed while maintaining the user fully space-aware.

Another advantage of HoloLens implementation is lack of problems with calibrating the display for children. Fullycovering VR setups are likely to present improper behavior when calibrated for children [14]. The device may lose the focus point, so the sight does not precisely follow the movements of user's head. Another advantageous feature of the HoloLens approach is the independent control of the contents displayed to each eye. Therefore, full control on the balance of the object projected for each eye is maintained and may be adjusted accordingly to the clinical diagnosis, to stimulate the lazy eye with different difficulty/intensity, while supporting the skill of stereo-vision.

Microsoft HoloLens supports streaming of the video displayed for user to any web browser. Thereby other persons may see the real-time cast of the holographic projection. This feature might be advantageous in supervision of the treatment process, as the doctor can dynamically monitor the patients activity. Patient can be therefore guided on specific actions performed within the solution, which is supportive for more complex exercise. Moreover, the clinician is able to assess the performance of the user in completion of the holographic tasks. Therefore the level of difficulty can be easily adapted to skills and abilities of the particular user. 
Furthermore, the ability of the HoloLens to record the displays throughout the holographic session might be an asset for improving the exercise scenarios for future patents and enable extensive and precise analysis of users' performance and the results of the therapy applied.

\section{THE EXERCISE APPLICATION}

A simple game has been implemented, in which the user moves a spacecraft trying to omit falling asteroids. The space ship reacts to the movements of user's head. Asteroids are falling down one set after another with constant speed. One can adjust number of asteroids in a single set and the time interval between them. The artifacts displayed have been divided accordingly to the importance and awareness requirements to the user.

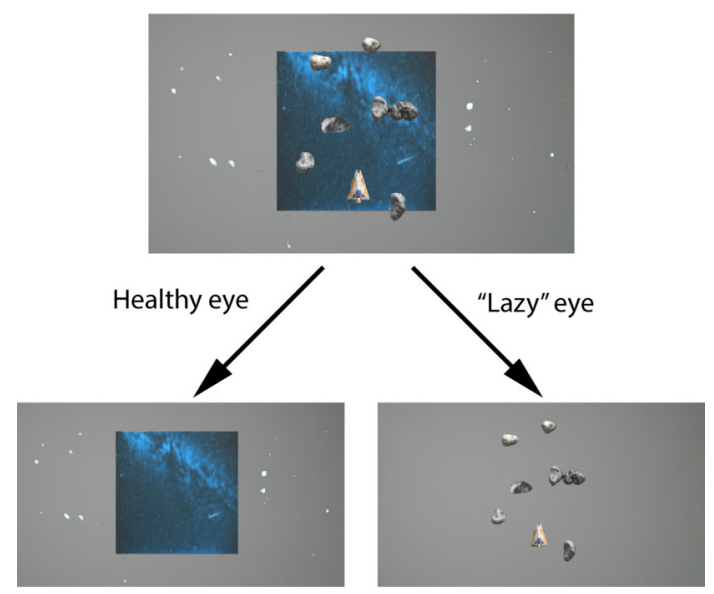

Fig. 2. Exemplary image displayed in the app. Left: the healthy eye part, containing the bacground. Right: the lazy eye part, containing roaming objects in the display.

All the important objects, such as spacecraft controlled and falling asteroids are displayed by single eye projection only the one set to operate for the amblyopic eye, whereas supplementary objects, such as background and navigation panel are displayed in the healthy-eye part. Therefore, the user's brain is forced to operate the lazy eye to participate in the game. The character of the game imposes high activity of the eye treated. Simultaneously, the healthy eye is not excluded from the perception, unlike in the traditional treatment approach. Therefore, the game acts as an exercise for restoring the proper stereo vision.

Moreover, the exercise is presented as a form of entertainment, which makes the treatment process more pleasant, encouraging to greater compliance to the prescribed training routine. Regarding that the lazy-eye syndrome therapy concerns mostly children, it is even more desirable to provide an enjoyable manner of exercising. Working version of the system was tested on 4 adults (38yo on average) and 4 children (6.5yo on average), including two patients with amblyopia; one in each group. All of the participants were rather new to mixed reality technology. After initial training (c.a. 5 min for adults and c.a. 2 min for children) participants were able to play the game successfully. Children reported more initial problems with starting as well as during the normal operation in the game, yet did not reported problems within the game itself. Adult users reported less problems with starting the game but assessed the play as difficult or requiring too quick reactions for them to follow. Amblyopia child assessed game to be more demanding in terms of both effort as well as initial training in comparison to his experience (very limited, yet wider) with VR Ocullus Rift version of Vivid Vision app. All of the adults as well as all of the children reported more comfort when using HoloLens comparing to VR experience. However, we cannot present any structured nor longitudinal study results so far neither in terms of app assessment nor in terms of therapeutic matters.

\section{Discussion}

The proposed solution employs recent findings in the lazyeye syndrome treatment in terms of using modern visual interfaces towards stimulating the disabled vision apparatus. Similar solutions have been offered before, while being aimed mostly for clinical or supervised use. Our system enables the participant to reach more self-reliance during the treatment and make the exercise procedure highly more convenient. The supervised mode is optional and requires additional device to which the sight from HoloLens could be casted.

The efficiency of the exercise is expected to be comparable to that obtained using traditional, full-covering VR approach. However, reduced number and intensity of possible side effects, like digestive discomforts, vertigo and labyrinth disorders is expected [15]. Due to limited projection range and the holographic technology applied, there is less risk of high eye-exhaustion or eye strain syndromes to occur [16]. The proposed solution is the next step towards providing the accessible and convenient tool for home-based amblyopia treatment-supporting procedures. The systems offers an entertaining manner of exercising the disabled eye, with additional activity monitoring features for enhanced supervision. The suggested approach lacks of common disadvantages of the arrangements used so far - those are the undesired side effects, inability to use the system for a long time, complex operation and/or difficult setting requirements.

\section{FUTURE WORK}

This paper presents a prototype software for the lazy-eye stimulation. Further improvements of the game interface; i.e. more entertainment/gamification features are planned in order to maintain interest of the user for long-period treatments. Additional context-aware sensing may be advisable, for precise assessment of the physical objects in sight and the distance between them and the player, so to obtain the system which could revise itself in order to provide as safety playing conditions as possible, which is a crucial issue concerning the children being a decent group within the target users. Furthermore, the device might be supplied with the gaze tracking system and help in controlling user interfaces [17] [18]. Regarding the efficiency 
of using the system in terms of clinical improvements in eye operation, it is necessary to provide a long-term study with a satisfactorily large sample and the control group to assess system's efficiency as a treatment tool. Its' operation is expected to be similarly effective as the VR-based solutions, with the extended support for stereo-vision development. Yet it is expected to overperform VR versions since AR technology is superior in terms of environment awareness and therefore lack of nausea effect and better suitability to young brains development. It would be interesting to employ this technology and similar app design to other settings such as the industrial environments, where similar features (distinct eye display mode) could be utilized for more efficient data analysis and flow instalations control parameters visualisation [19] [20] [21] [22]. The approach may also become useful considering modern visualisation systems for control purposes [23]. [24]. Perhaps most interesting and challenging would be to use the AR technology to implement in hybrid systems such as context-aware data processing or crowdsourcing applications [25] [26] [27].

\section{CONCLUSIONS}

This work shows a new approach to using computer vision technology for amblyopia therapy. Namely, mixed reality paradigm is applied in order to overcome disadvantages of virtual reality displays that already proven their potential for lazy eye treatment. Therefore, the proposed approach using Hololens device enables to exercise the weaker eye without full immersing into the virtual world. This is of a key advantage over the VR technology especially for children under 14 due to immature nervous system, middle ear bone labyrinth and eye fundus development.

\section{REFERENCES}

[1] J. M. Holmes and M. P. Clarke, “Amblyopia," The Lancet, vol. 367, no. 9519 , pp. 1343 - 1351, 2006.

[2] P. Waddingham, S. Cobb, R. Eastgate, and R. Gregson, "Virtual reality for interactive bi nocular treatment of amblyopia," in Proc. 6th Intl Conf. Disability, Virtual Reality \& Assoc. Tech., Esbjerg, Denmark, 2006.

[3] B. Thompson, B. Mansouri, L. Koski, and R. Hess, "Brain plasticity in the adult: Modulation of function in amblyopia with rtms," Current Biology, vol. 18, Issue 14, 2008.

[4] R. F. Hess, B. Mansouri, and B. Thompson, "A new binocular approach to the treatment of amblyopia in adults well beyond the critical period of visual development," Restorative Neurology and Neuroscience 28 1-10, 2010.

[5] R. Li, C. Ngo, and D. L. J. Nguyen, "Video-game play induces plasticity in the visual system of adults with amblyopia," PLoS Biol 9(8), 2011.

[6] R. Eastgate, "Modified virtual reality technology for treatment of amblyopia," Eye volume 20, pages 370-374, 2006.

[7] P. Žiak, A. Holm, J. Halička, P. Mojžiš, and D. P. Piñero, "Amblyopia treatment of adults with dichoptic training using the virtual reality oculus rift head mounted display: preliminary results," BMC Ophthalmology, vol. 17, p. 105, Jun 2017.

[10] J. Blaha, "Initial study results indicate vr game is effective in improving vision in people with lazy eye," 2015.
[8] E. Birch, "Binocular ipad treatment for amblyopia in preschool children," Journal of American Association for Pediatric Ophthalmology and Strabismus JAAPOS , Volume 19, Issue 1, 6 - 11, 2014.

[9] A. Gargantini, "A low-cost virtual reality game for amblyopia rehabilitation," in REHAB '15 Proceedings of the 3rd 2015 Workshop on ICTs for improving Patients Rehabilitation Research Techniques, 2015.

[11] D. Mezad-Koursh, "Home use of binocular dichoptic video content device for treatment of amblyopia: a pilot study," Journal of American Association for Pediatric Ophthalmology and Strabismus JAAPOS, 2016.

[12] M. Meehan, B. Insko, M. Whitton, and F. P. Brooks, Jr., "Physiological measures of presence in stressful virtual environments," ACM Trans. Graph., vol. 21, pp. 645-652, July 2002.

[13] M. S. Maria V. Sanchez-Vives, "From presence to consciousness through virtual reality," Nature Reviews Neuroscience volume 6, pages 332-339, 2005.

[14] Z. Pan, A. D. Cheok, H. Yang, J. Zhu, and J. Shi, "Virtual reality and mixed reality for virtual learning environments," Computers \& Graphics, vol. 30, no. 1, pp. $20-28,2006$.

[15] P. C. Grigore C. Burdea, Virtual Reality Techology. 2003.

[16] F. L. Kooi and A. Toet, "Visual comfort of binocular and 3d displays," Displays, vol. 25, no. 2, pp. 99 - 108, 2004.

[17] A. Wojciechowski and K. Fornalczyk, "Exponentially smoothed interactive gaze tracking method.," Springer, Cham, In International Conference on Computer Vision and Graphics (pp. 645-652), 2014.

[18] G. Glonek and A.Wojciechowski, "Hybrid orientation based human limbs motion tracking method," Sensors, vol. 17(12), 2017.

[19] A. Romanowski, "Big data-driven contextual processing methods for electrical capacitance tomography," IEEE Transactions on Industrial Informatics, vol. doi:10.1109/TII.2018.2855200, p. in press, 2018.

[20] K.Grudzien, A.Romanowski, D.Sankowski, and R.A.Williams, "Gravitational granular flow dynamics study based on tomographic data processing," Particulate Science and Technology, vol. 26, no. 1, pp. 67$82,2008$.

[21] V. Mosorov and D. Sankowski, "Estimation of the rotation angle of gas/solid swirl flow by subpixel image resizing," Asia-Pacific Journal of Chemical Engineering, vol. 13, no. 2, p. e2177, 2018.

[22] K. Grudzien, "Visualization system for large-scale silo flow monitoring based on ect technique," IEEE Sensors Journal, vol. 17, pp. 8242-8250, December 2017.

[23] M. Woźniak, A. Polak-Sopińska, A. Romanowski, K. Grudzień, Z. Chaniecki, A. Kowalska, and M. Wróbel-Lachowska, "Beyond imaging - interactive tabletop system for tomographic data visualization and analysis," in Advances in Manufacturing, Production Management and Process Control (W. Karwowski, S. Trzcielinski, B. Mrugalska, M. Di Nicolantonio, and E. Rossi, eds.), (Cham), pp. 90-100, Springer International Publishing, 2018.

[24] A. Romanowski, K. Grudzien, Z. Chaniecki, and P. Wozniak, "Contextual processing of ECT measurement information towards detection of process emergency states," in Hybrid Intelligent Systems (HIS), 2013 13th International Conference on, pp. 291-297, 2013.

[25] C. Chen, P. W. Woźniak, A. Romanowski, M. Obaid, T. Jaworski, J. Kucharski, K. Grudzień, S. Zhao, and M. Fjeld, "Using crowdsourcing for scientific analysis of industrial tomographic images," ACM Trans. Intell. Syst. Technol., vol. 7, no. 4, pp. 52:1-52:25, 2016.

[26] I. Jelliti, A. Romanowski, and K. Grudzien, "Design of crowdsourcing system for analysis of gravitational flow using x-ray visualization," in FedCSIS'16, ACSIS, vol. 8. IEEE, p. 1613-1619.

[27] A. Romanowski, "Contextual processing of electrical capacitance tomography measurement data for temporal modeling of pneumatic conveying process," in Proceedings of the 2018 Federated Conference on Computer Science and Information Systems, FedCSIS'18, ACSIS, vol. IEEE, p. in press. 